\title{
PAPR Reduction in OFDM System Using Clipping and Filtering Methods Based on CCDF
}

\author{
Md. Tanvir UI Haque and Md. Sharif Hossen \\ Dept. of Information and Communication Technology Comilla University, Bangladesh

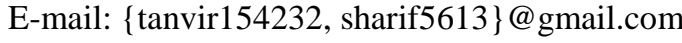

Received: 17 May 2019; Accepted: 16 July 2019; Published: 08 October 2019

\begin{abstract}
Wireless communication systems are becoming so promising day-by-day due to the mobility and the dynamicity of communication pattern. But, to fulfill the wide range of user's demand it has become much important to use some techniques which would be most efficient in terms of bandwidth and speed. The multicarrier strategy, called as orthogonal frequency division multiplexing (OFDM) has outstanding features to fulfill these demands. Multipath fading, delay spread, frequency selective fading and inter channel interference all of these limitations of wireless communication compound it with the scarcity of bandwidth gave rise to OFDM. However, the high peak-to-average power ratio is a great problem or a barrier in OFDM which causes the signal being distorted with the insufficient power at the receiver. There are some specified techniques to minimize it. In this paper, we have used clipping and filtering methods to minimize the effect of peak-toaverage power ratio.
\end{abstract}

Index Terms-OFDM, PAPR, Fourier transform, Clipping, and Filtering.

\section{INTRODUCTION}

In recent years, wireless communications are getting more improvements in a very speedy way due to the growing demand of wireless services [1]. Since wireless channels cannot be perfectly predictable, it is slightly difficult to find the noise and the propagation of signal. While, in wired channels, the propagation of signal follows a predefined path, and so the noise is easily calculated in the channel. Using AWGN as a channel in the communication system tends to reduce the signal strength which makes the signal being distorted for the sources which may be industrial or natural [2]. Fading channels can be of different types like multipath, and frequency selective. Transmission of signal can be possible through a single path and multipath. Signal can be transmitted through a single carrier called single carrier transmission technique which is much easier to use for its simplicity. The main problem of using it is to take the high transmission time with a chance of complete loss of full transmission. But, using multicarrier transmission, the possibility of appearing multipath fading is high which causes the inter symbol interference due to the interference of symbols. As a multicarrier technique we use orthogonal frequency division multiplexing (OFDM) to defeat the problem of single carrier so that high date rate is achievable [3, 4]. The remaining part of this paper is arranged in the following way: In section 2, we discuss the principle of OFDM system with its benefits, and problems. Section 3 includes the discussion of peak-to-average power ratio with its characteristics and complementary CDF. In section 4, we see the classification of strategies used to minimize the PAPR and discuss the considered methods used in this paper. In section 5, we discuss the step by step experimental results with proper arguments. Finally, section 6 includes the research summary with upcoming works.

\section{PRINCIPLE OF OFDM WITH MERITS AND DEMERITS}

The background behind OFDM is that it divides the frequency spectrum into subcarriers which are mutually independent to each other, i.e., orthogonal so that interference between symbols is minimized. The data in each subcarrier are transformed from serial to parallel channel for simultaneous transmission in different channel. The inverse fast fourier transform, i.e., IFFT, can be utilized to generate the orthogonal features where either QAM or BPSK are used to modulate the sample data and then the modulated results are correlated jointly [5]. The function of FFT is to transform the time representation into frequency. OFDM has significant challenge due to the symbol time less than the delay spread leading to ISI which can be minimized by using cyclic prefix or guard band concept [6,7]. The basic structure of OFDM is shown in Figure 1.

From the above figure we see that at first the modulating signal $\mathrm{s}(\mathrm{t})$ is passed through a serial to parallel converter to transform the serial stream into a parallel form. The parallel streams are then passed through an IFFT block which is then passed through a cyclic prefix to add each part with a cyclic block. Then, the output of cyclic prefix block is converted into serial stream by passing through a parallel to serial converter block which is then passed through the transmitter. After that the serial stream are passed through the channel. The received signal streams are then passed through Receiver filter. After that, it is passed through $\mathrm{S} / \mathrm{P}$, cyclic prefix 
removal, FFT, P/S block. Finally, the received signal which is sent from the sender end is received by the receiver.

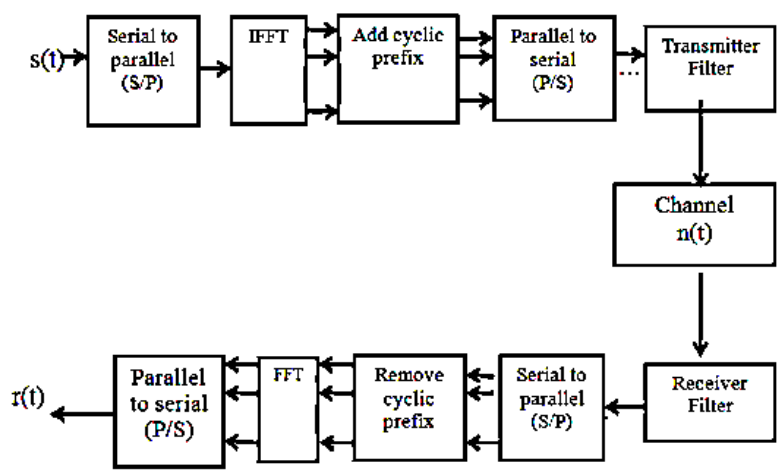

Fig.1. Basic Structure of OFDM system.

In the communication system, to solve the problem of single carrier, OFDM multicarrier is used which provides very high data rates by reducing the effects of multipath and ensuring the proper use of frequency spectrum [8]. The problem with OFDM is to rise the high PAPR due to the use of nonlinear amplifier which degrades the results of output amplifier. An OFDM signal is basically generated by the combination of many independent subcarriers after modulation which makes high peak and thereby resulting high PAPR [9]. This finally introduces the distortion and high bit error. Many techniques have been discovered to recover the PAPR effect $[10,11,12]$.

OFDM system offers the following merits [13]:

1. High spectral efficiency

2. Lower multi-path distortion

3. Spectrum can be used perfectly by supporting the overlapping symbols

4. Reduction of ISI has been possible by the inclusion of cyclic prefix

5. Computationally efficient implementation of the demodulation and modulation processes.

It has some limitations. These are:

1. Sensitive to frequency offset

2. Large peak to average ratio

3. IFFT/FFT complexity

4. Inter-symbol interference (ISI) due to multipath

\section{PAPR AND CCDF}

The most challenging problem in the communication system is very high PAPR [15]. The main reason of having it is the presence of irregular power spectrum that means we need to keep the symbol stream of IFFT uniform [5, 16, 17]. Another reason of having high PAPR is to use the huge amount of subcarriers which causes some difficulties at the end of transmission [18, 19, 20]. We can define PAPR as the ratio of the peak or maximum power during the transmission of OFDM symbol with respect to its mean power. Mathematically, it is as follows:

$$
P A P R=\frac{\max [|x(n)|]^{2}}{E\left[\left|x(n)^{2}\right|\right]}
$$

where $|x(n)|$ is the value $x(n)$ and the expected value of it is $E[]$.

A peak is generated by the combination of $\mathrm{N}$ signals with equal phase [21]. With $\mathrm{N}$ subcarriers, we can get a peak value of utmost $10 \log \mathrm{N}(\mathrm{dB})$.

Now, we will discuss the effects of PAPR as shown in Figure 2. Let us consider the amplifier characteristics. The linear amplifier range of a device exists only a certain range. If the input is larger than the voltage range, the output is no longer linear. It becomes linear that means saturated $[22,23]$.

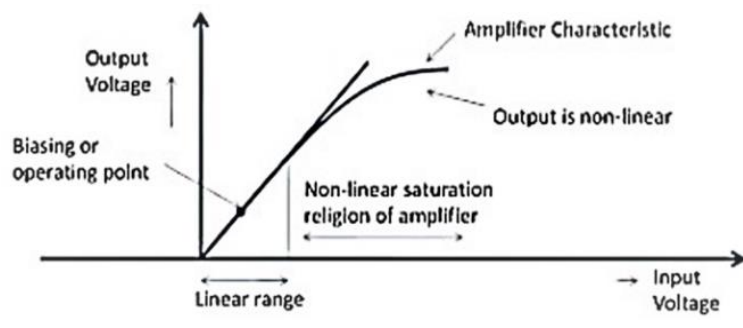

Fig.2. Amplifier characteristics.

The problem in the OFDM [28] system is to have nonideal amplifier in practice. It has a limited linear amplification range and the swing of the peak power can be greater than the expected power. Here, the amplifier crosses over the saturation region. The output is no longer linearly related to the input, means that OFDM properties are sized. So, orthogonality is lost, and inter carrier interference (ICI) is introduced [6]. We can use complementary CDF to determine the performance of each PAPR strategy. The main reason of using CCDF rather than $\mathrm{CDF}$ is to get that possibility of a data block which is greater than a fixed value [24, 25]. Using a greater amount of subscribers for such a system, we consider that the real and imaginary components contain a mean of 0 with a variance of $1 / 2$ which introduces the distribution of Gaussian. The equation of a CDF of such a signal amplitude is given by

$$
f(x)=1-\exp (x)
$$

After calculating CDF, we can determine the CCDF of each block of PAPR as follows-

$$
\begin{aligned}
P(P A P R>x) & =1-P(P A P R \leq x) \\
& =1-f(x)^{N} \\
& =1-(1-\exp (-x))^{N}
\end{aligned}
$$

\section{CLIPPING AND FILTERING}

In recent years, a number of strategies have been proposed to minimize the PAPR [26, 14]. Some components before implementing the filtering and clipping strategies are essential to maintain the system 
requirements which are greater efficiency of spectrum, to prevent the degradation of data rate and signal strength, lower bit error, etc. These reduction strategies are classified into two broad categories, namely, signal distortion and scrambling which are shown in Figure 3.

The simplest strategy is clipping where a high signal voltage exists before traveling it across the specified amplifier. Using this, we can minimize the power by adjusting an utmost point of the signal being sent [27]. However, it faces few problems as follows:

1. High amount of in-band distortion can change BER reversely.

2. Adjacent interference can be found due to the outof-band distortion.

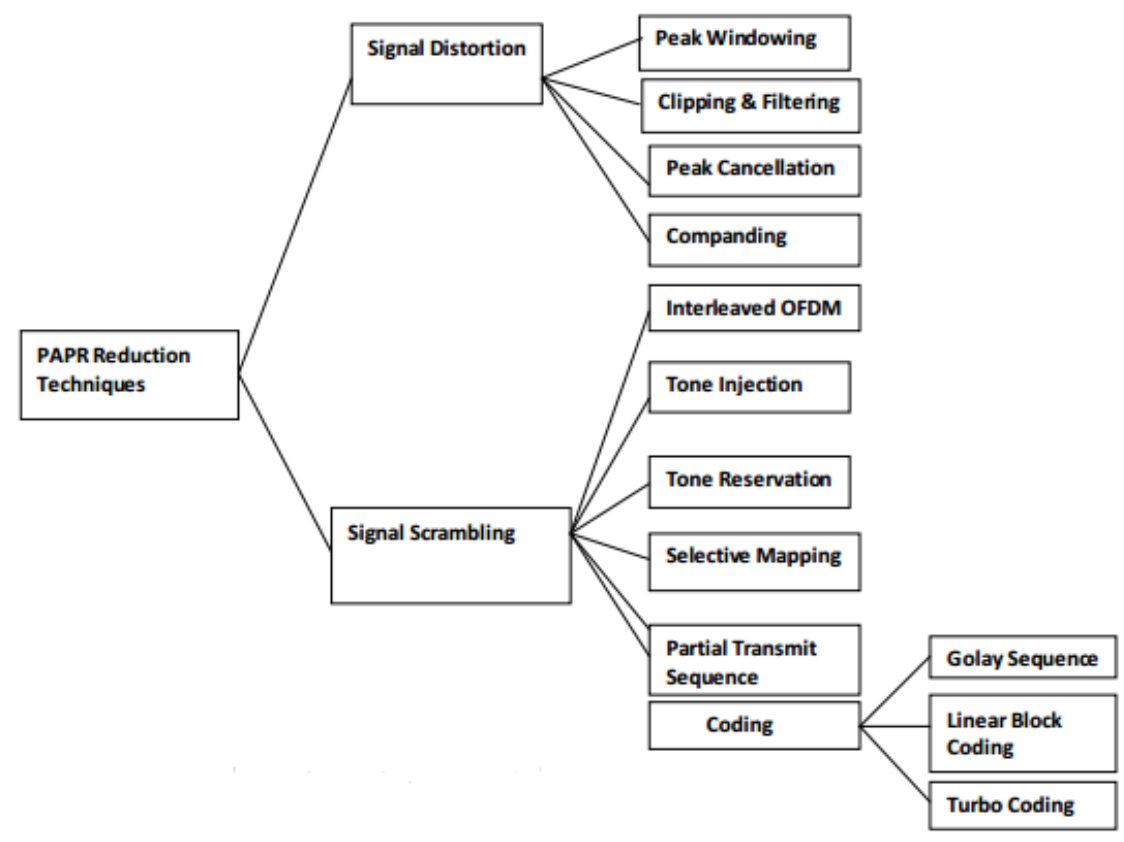

Fig.3. PAPR Minimization Strategies.

The distortion mentioned in (ii) can be reduced by using filtering techniques where there will be a high possibility of passing the utmost point of the clipping strategy. The basic structure of the considered strategies in this paper is shown in Figure 4. Here, at first the signal are passed through a L-N point IFFT block where total subcarrier and oversample components are indicated by respective symbols $\mathrm{N}$ and $\mathrm{L}$. This block produces L-times output which is passed through digital up conversion (adding carrier frequency here) block whose output is then clipped. The resulting output is then passed through L-N point FFT, BPF, again L-N point IFFT and finally LPF. Less BER and distortion is achieved at the output of filtering. The signal before BPF (clipping level indicated by $\mathrm{B}$ ) is expressed as follows:

$$
x_{c}^{p}[m]=\left\{\begin{array}{cc}
-B & x^{p}[m] \leq B \\
x^{p}[m] & \left|x^{p}[m]\right|<B \\
B & x^{p}[m] \geq B
\end{array}\right.
$$

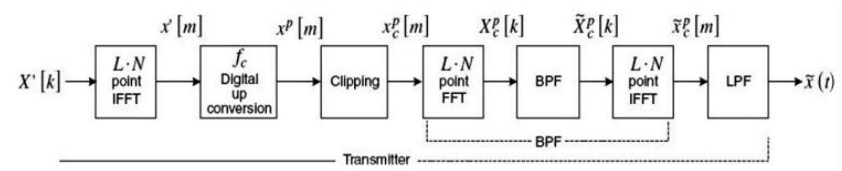

\section{RESULT AND DISCUSSION}

We use Matlab as the simulation tool. In the experimental figures, we show the original signal of OFDM and also the signal which is clipped and filtered. We also plot CCDF comparison between original, clipped and filtered OFDM signals.

Table 1. Arguments for clipping and filtering

\begin{tabular}{|c|c|}
\hline Arguments & Value \\
\hline Sub-carriers (N) & $64,128,256,512,1024$ \\
\hline Modulation & 16-QAM \\
\hline Oversampling Factor (L) & 4 \\
\hline Clipping level & 0.70 \\
\hline Channel Model & AWGN \\
\hline
\end{tabular}

Using $\mathrm{N}=64$, Figure 5 (b) indicates that without clipping, PAPR is $11.5 \mathrm{~dB}$ at CCDF of $10^{-4}$. PAPR is reduced to $8.9 \mathrm{~dB}$ after clipping but $6.5 \mathrm{~dB}$ after applying clipping and filtering techniques. The $\mathrm{CF}$ procedure achieves PAPR reduction by $43.47 \%$ when compared to the original OFDM signal but by clipping, it achieves only $22.60 \%$.

Fig.4. PAPR reduction scheme using clipping and filtering. 


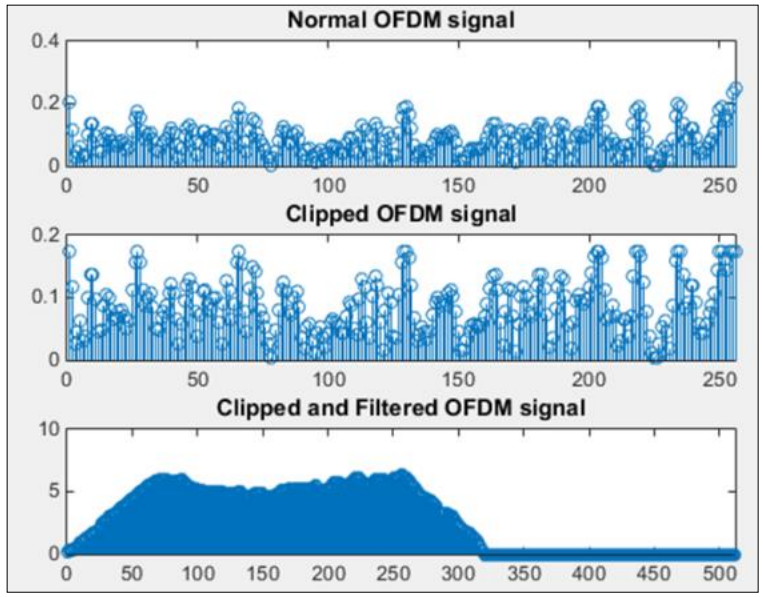

(a) Signals

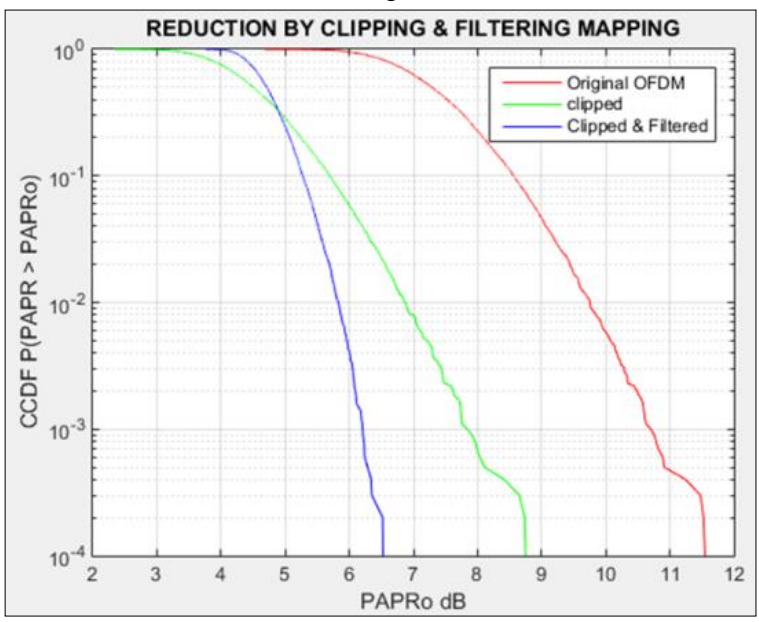

(b) $\mathrm{CCDF}$

Fig.5. Representation of (a) signals and (b) CCDF comparison for $\mathrm{N}=64$

Figure 6 (a) shows the OFDM signal using $\mathrm{N}=128$. Figure 6 (b) indicates without clipping, PAPR is $11.4 \mathrm{~dB}$ at $\mathrm{CCDF}$ of $10^{-4}$. PAPR is reduced to $8.6 \mathrm{~dB}$ after clipping but $5.7 \mathrm{~dB}$ after applying clipping and filtering technique. The CF procedure achieves PAPR reduction by $50 \%$ when compared to the original OFDM signal but by clipping, it achieves only $24.13 \%$.

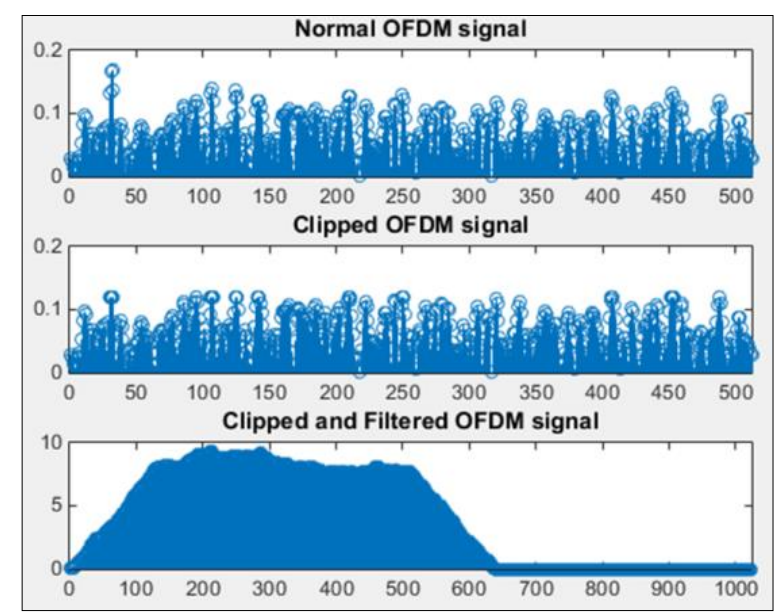

(a) OFDM signal

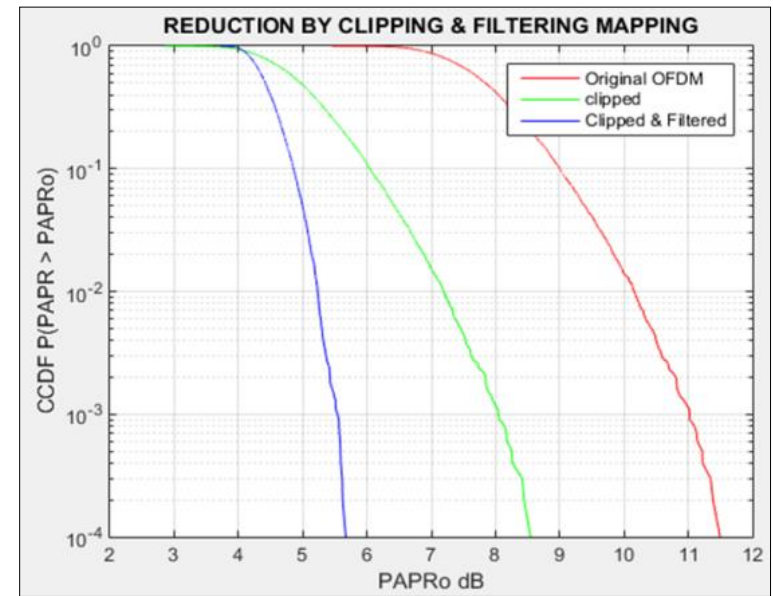

(b) $\mathrm{CCDF}$

Fig.6. Representation of (a) signals and (b) CCDF comparison for $\mathrm{N}=128$.

Figure 7 (b) indicates that without clipping, PAPR is $11.98 \mathrm{~dB}$ at CCDF of $10^{-4}$. PAPR is reduced to $8.9 \mathrm{~dB}$ after clipping but $5.3 \mathrm{~dB}$ after applying clipping and filtering techniques. The CF procedure achieves PAPR reduction by $55.75 \%$ compared to the original OFDM signal but by clipping it achieves only $25.70 \%$.

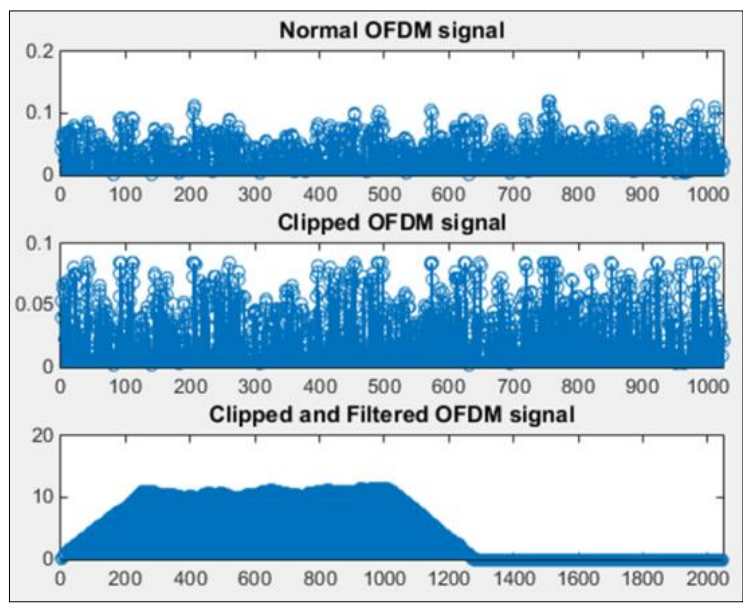

(a) OFDM Signal

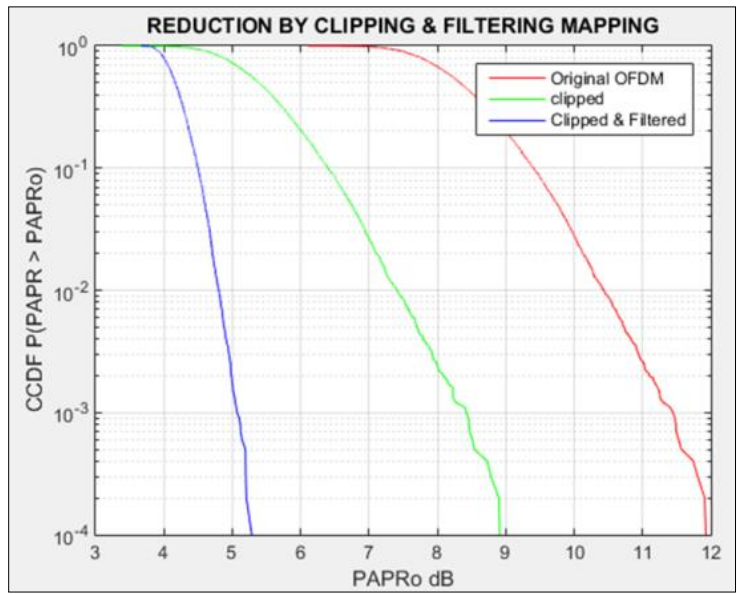

(b) $\mathrm{CCDF}$

Fig.7. Representation of (a) signals and (b) CCDF comparison for $\mathrm{N}=256$. 
Figure 8 (b) indicates that without clipping, PAPR is $11.99 \mathrm{~dB}$ at CCDF of $10^{-4}$. PAPR is reduced to $8.8 \mathrm{~dB}$ after clipping but $4.6 \mathrm{~dB}$ after applying clipping and filtering technique. The CF procedure achieves PAPR reduction by $61.63 \%$ when compared to the original OFDM signal but by clipping, it achieves only $26.60 \%$.

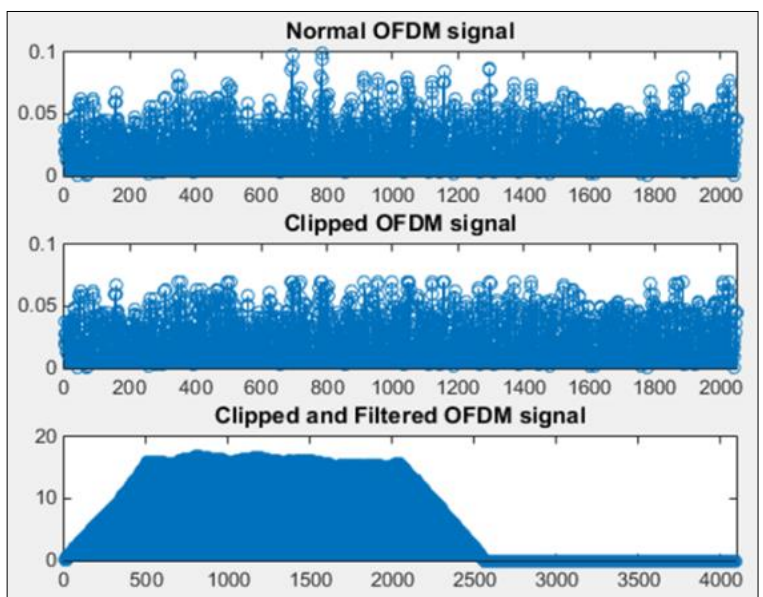

(a) OFDM Signal

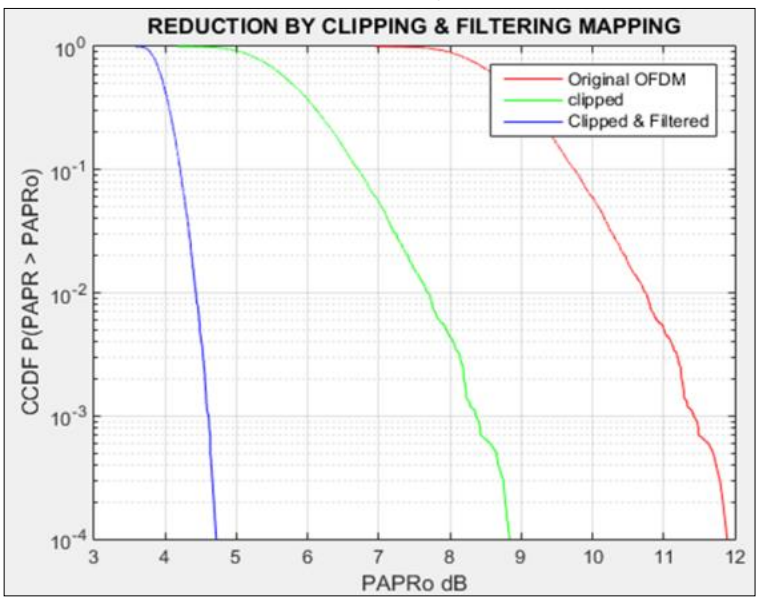

(b) $\mathrm{CCDF}$

Fig.8. Representation of (a) signals and (b) CCDF comparison for $\mathrm{N}=512$.

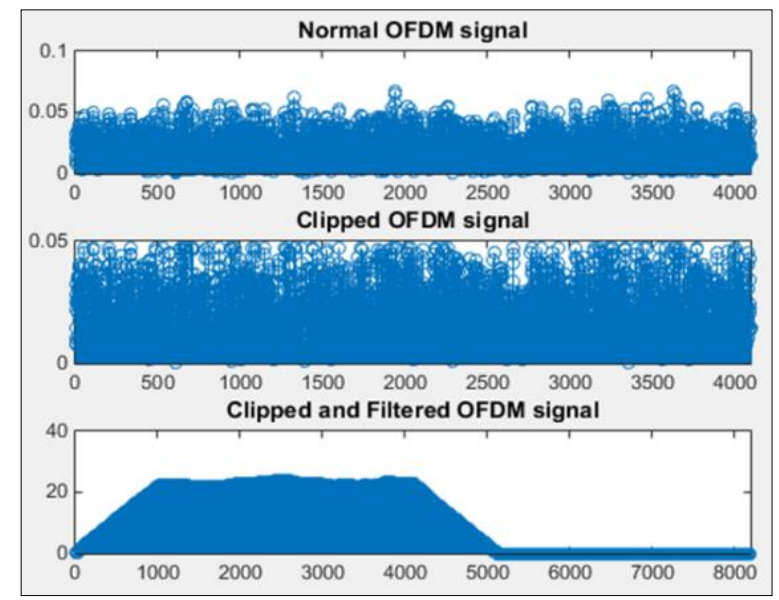

(a) OFDM Signal

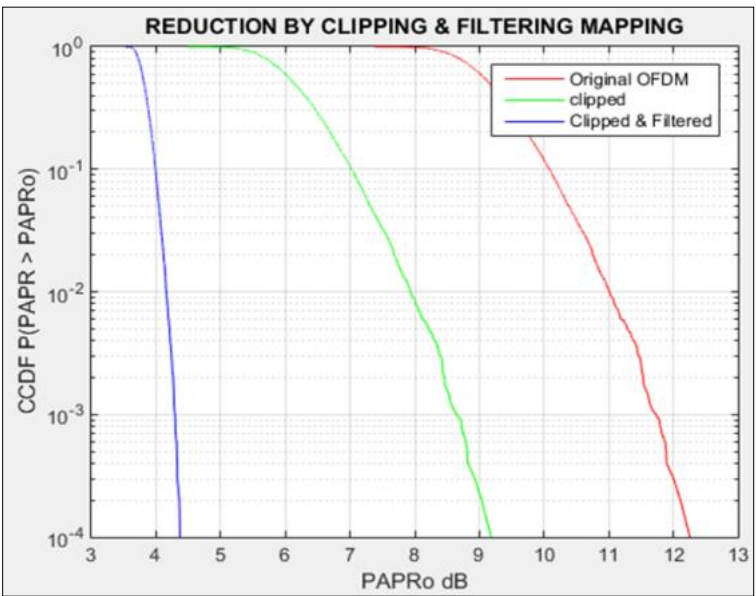

(b) $\mathrm{CCDF}$

Fig.9. Representation of (a) signals and (b) CCDF comparison for $\mathrm{N}=1024$.

Figure 9 (b) indicates that without clipping, PAPR is $12.2 \mathrm{~dB}$ at CCDF of $10^{-4}$. PAPR is reduced to $9.1 \mathrm{~dB}$ after clipping but $4.3 \mathrm{~dB}$ after applying clipping and filtering technique. The $\mathrm{CF}$ procedure achieves PAPR reduction by $64.47 \%$ when compared to the original OFDM signal but by clipping, it achieves only $25.54 \%$.

\section{CONCLUSION AND FUTURE WORKS}

Orthogonal frequency division multiplexing (OFDM) is a multicarrier strategy where a broadband channel is divided into multiple narrowband sub-channels, i.e., a high speed data rate stream is divided into many parallel low data rate stream which uses the respective subchannels instead of using a single broadband channel. The major problem in OFDM system is high peak-toaverage power ratio (PAPR). In this paper, we have attempted to minimize the PAPR using clipping and filtering strategy on CCDF. Our investigation indicate how these strategies improve upon the PAPR of OFDM signal where clipping level contributes to clip a signal while distortion is reduced with the use of filtering. With the increase of subcarriers, the investigated results of CCDF vary also. Using clipping and filtering strategies, we observe lower $\mathrm{CCDF}$ for $\mathrm{N}=1024$ compared to using other subcarriers, $\mathrm{N}$.

In near future, we would like to improve the performance by minimizing lower PAPR with a hybrid technology which is the combination of clipping and SLM technique.

\section{ACKNOWLEDGMENT}

The authors would like to thank the anonymous reviewers to carefully review the article. Besides, they wish to thank the Editor. 


\section{REFERENCES}

[1] John, A. (1990), "Multicarrier modulation for data transmission: an idea whose time has come," IEEE Communications Magazine.

[2] Albdran et al. (2012), "The sensitivity of bit error rate (BER) performance in multi-carrier (OFDM) and singlecarrier," SPIE optics and photonics.

[3] Wu, Y. \& Zou, W.Y. (1995), "Orthogonal frequency division multiplexing: A multi-carrier modulation scheme," IEEE Trans. Consumer Electronics, vol. 41, no. 3, pp. 392- 399

[4] Taub et al. (2008), "Taub's principles of communication systems," McGraw Hill.

[5] Nee, R. V. \& Prasad, R. (2000), "OFDM wireless multimedia communications," Artech House, Boston.

[6] Han, S. H. \& Lee, J. H. (2005), “An overview of peak-toaverage power ratio reduction techniques for multicarrier transmission," IEEE Wireless Communication, vol. 12, no 2, pp. 56-65.

[7] Ochiai, H. \& Imai, K. (2000), "Clipping for peak power reduction of OFDM signals," IEEE GTC, vol. 2, pp. 731735.

[8] Chu, D.C. (1972), "Polyphase codes with periodic correlation properties," IEEE Trans. Info. Theory, 18(4), 531-532.

[9] H.D. Luke, and H.D. Schotten, and H.H. Mahram, "Binary and quadric-phase sequences with optimal autocorrelation properties," IEEE Trans. Info. Theory, 49(12), 3271-3282, 2003

[10] Y. Rahmatallah, and S. Mohan, "Peak-to-average power ratio reduction in OFDM schemes: a survey and taxonomy," IEEE Communications Surveys and Tutorials, vol. 15 , no. $4,2013$.

[11] S. H. Muller, and J.B. Huber, "A comparison of peak power reduction schemes for OFDM," IEEE Global Communications Conference, Phoenix, AZ, pp. 1-5, Nov. 1997.

[12] Banelli, P. (2011), "Another Useful Theorem for NonLinear Transformations of Gaussian Random Variables,"

[13] Jiang, T. \& Wu Y. (2008), "An Overview: Peak-toAverage Power Ratio Reduction Techniques for OFDM Signals," IEEE Transactions on Broadcasting, vol. 54, pp. 257-268.

[14] Jeon et al. (2012), "A New PAPR Reduction Scheme Using Efficient Peak Cancellation for OFDM Systems," IEEE Trans Broadcast, vol. 58(4), pp. 619-628.

[15] Urban J., \& Marsalek R. (2007), "OFDM PAPR Reduction by Combination of Interleaving with Clipping and Filtering," IEEE Communication Letter, pp. 249- 252.

[16] Yao et al. (2013) "OFDM System with Reduce Peak-toAverage Power Ratio Using Optimum Combination of Partial Transmit Sequences," In: Tan Y., Shi Y., Mo H. (eds) Advances in Swarm Intelligence. Lecture Notes in Computer Science, vol 7928. Springer, Berlin, Heidelberg.

[17] Chen L. \& Hu, X. (2009), "Peak-to-Average Power Ratio Reduction of an OFDM Signal Using Signal Scrambling," $2^{\text {nd }}$ International Congress on Image and Signal Processing, IEEE publisher, Tianjin, China.

[18] Sumithra, M. G., \& Sarumathi, M. (2011), "PAPR Reduction Techniques for Multicarrier Systems," Global Trends in Information Systems and Software Applications. Communications in Computer and Information Science, vol. 270. Springer, Berlin, Heidelberg.

[19] R.W. Bauml, R. F. H. Fischer, and J.B. Huber, "Reducing the peak-to-average power ratio of multicarrier modulation by selected mapping," IEEE Electronics Letters, vol. 32, pp. 2056-2057, 24 Oct. 1996.

[20] Fischer, R. F. H. (2007), "Widely-Linear Selected Mapping for Peak-To- Average Power Ratio Reduction in OFDM," IEEE Electronics Letters, pp. 766-767.

[21] Alhasson, B. \& Matin M. (2011), "Reduction of PAPR for OFDM Downlink and IFDMA Uplink Wireless Transmissions," International Journal of Computer Science and Information Security.

[22] Saini, S. \& Sahu, O. P. (2012), "Peak-to-Average Power Ratio Reduction in OFDM System by Clipping and Filtering," International Journal of Electronics Communication and Computer Technology (IJECCT), vol 2 , Issue 3

[23] Jha, \& Shanker, U. (2007), "OFDM Towards Broadband Wireless Access", Artech House Books, Norwood.

[24] Quadrature Amplitude Modulation, https://en.wikipedia.org/wiki/Quadrature_amplitude_mod ulation (accessed February 26, 2019).

[25] Oversampling, https://en.wikipedia.org/wiki/Oversampling (accessed February 26, 2019).

[26] J. Hou, J. Ge, and J. Li, "Peak-to-average power ratio reduction of OFDM signals using PTS scheme with low computational complexity," IEEE Transactions On Broadcasting, vol. 57, pp. 143-148, March 2011.

[27] L. Yang, Y. M. Siu, K. K. Soo, S. W. Leung and S.Q. Li, "Low complexity PAPR reduction technique for OFDM systems using modifed widely linear SLM scheme," Int. J. Electron. Commun., pp. 1006-1010, May 2012.

[28] A. E. Jones, T. A. Wilkinson, and S. K. Barton. "Block coding scheme for reduction of peak to mean envelope power ratio of multicarrier transmission schemes," Electronics Letters, vol. 30(25), 1994.

[29] M. S. Hossen, and S. Ahmed, "Development of a Java Based Simulator for OFDM System," ICMEIE, University of Rajshahi, Bangladesh (2015).

\section{Authors' Profiles}

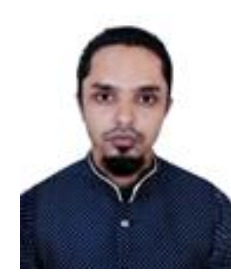

Md. Tanvir Ul Haque is a M.Sc. Student in the Department of Information and Communication Technology (ICT) at Comilla University (CoU), Bangladesh

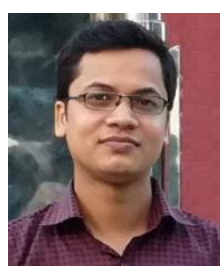

Md. Sharif Hossen is working as a Lecturer in the Department of Information and Communication Technology (ICT) at Comilla University (CoU), Bangladesh. He was formerly Lecturer in Department of Computer Science and Engineering (CSE) at Southeast University (SEU), and research assistant in Department of Information and Communication Engineering (ICE) at University of Rajshahi (RU). He completed his B. Sc. (Bachelor of Science) and M. Sc. (Master of Science) from Dept. of ICE at University of Rajshahi, Bangladesh. He achieved faculty first position in both degrees. His research interests include delaytolerant networking, wireless ad hoc, sensor, underwater and 
vehicular networks, as well as considerations of privacy in the Internet of Things. He is also interested in applying big data techniques. He has seven international journal papers (viz. Scopus Index, Springer), thirteen international conference papers (viz. IEEE, Springer) and one book chapter (viz. Taylor and Francis). He received several scholarships like ICT research fellowship (for M. Sc. thesis), UGC scholarship (for B. Sc.), and Merit scholarship for outstanding academic result at University of Rajshahi. He received Best Paper Award at IEEE ICISET 2016 conference at IIUC, Bangladesh and at Springer ICACIE 2018 conference at SOA University, India. He is the reviewer of several international journals (viz. Wireless Personal Communications, Springer).

How to cite this paper: Tanvir Ul Haque, Sharif Hossen, "PAPR Reduction in OFDM System Using Clipping and Filtering Methods Based on CCDF", International Journal of Computer Network and Information Security(IJCNIS), Vol.11, No.10, pp.12-18, 2019. DOI: 10.5815/ijcnis.2019.10.02 